\title{
ARTICLE
}

Received 23 Jan 2015 | Accepted 8 Sep 2015 | Published 22 Oct 2015

DOI: $10.1038 /$ ncomms 9585

OPEN

\section{Observation of a two-dimensional liquid of Fröhlich polarons at the bare $\mathrm{SrTiO}_{3}$ surface}

Chaoyu Chen ${ }^{1}$, José Avila ${ }^{1}$, Emmanouil Frantzeskakis ${ }^{1}{ }^{\dagger}$, Anna Levy ${ }^{1, \dagger}$ \& Maria C. Asensio ${ }^{1}$

The polaron is a quasi-particle formed by a conduction electron (or hole) together with its self-induced polarization in a polar semiconductor or an ionic crystal. Among various polarizable examples of complex oxides, strontium titanate $\left(\mathrm{SrTiO}_{3}\right)$ is one of the most studied. Here we examine the carrier type and the interplay of inner degrees of freedom (for example, charge, lattice, orbital) in $\mathrm{SrTiO}_{3}$. We report the experimental observation of Fröhlich polarons, or large polarons, at the bare $\mathrm{SrTiO}_{3}$ surface prepared by vacuum annealing. Systematic analyses of angle-resolved photoemission spectroscopy and X-ray absorption spectra show that these Fröhlich polarons are two-dimensional and only exist with inversion symmetry breaking by two-dimensional oxygen vacancies. Our discovery provides a rare solvable field theoretical model, and suggests the relevance of large (bi)polarons for superconductivity in perovskite oxides, as well as in high-temperature superconductors.

\footnotetext{
${ }^{1}$ Synchrotron SOLEIL, Beamline ANTARES, L'Orme des Merisiers, Saint Aubin-BP 48, Gif surYvette 91192, France. † Present addresses: Institute of Physics (loP), University of Amsterdam, Science Park 904, 1098 XH Amsterdam, The Netherlands (E.F.); Sorbonne Universits, UPMC, Paris 06, CNRS, INSP, UMR
} 7588, INSP, F-75005 Paris, France (A.L.). Correspondence and requests for materials should be addressed to M.C.A. (email: asensio@synchrotron-soleil.fr). 
T he concept of a polaron was set forth by Landau in 1933 and has attracted much attention over the following decades. It not only describes the specific physical properties of charge carriers in polarizable solid, but also constitutes an interesting field theoretical model consisting of a fermion interacting with a scalar boson field ${ }^{2}$. Even today, there is ongoing research on polarons, including the basic theory ${ }^{3}$, as well as their behaviour in more compelling situations such as hightemperature superconductors ${ }^{4-6}$ and colossal magnetoresistance in rare-earth manganites ${ }^{7}$.

There are different types of polaron states in solids, such as Fröhlich polaron, small polaron, spin polaron, bipolaron and hydrated polaron ${ }^{2}$. Originally polaron describes interaction between charge carrier and long-wavelength optical phonons, namely, Fröhlich polaron ${ }^{8}$, of which the spatial extension exceeds the lattice constant. Fröhlich polarons with low kinetic energy propagate through the lattice as free electrons but with an enhanced effective mass, thus directly measurable by spectroscopies like angle-resolved photoemission spectroscopy (ARPES).

$\mathrm{SrTiO}_{3}$ is the key material in the emerging field of oxide electronics ${ }^{9}$. Structurally or electronically modified $\mathrm{SrTiO}_{3}$ presents a wide spectrum of phenomena such as superconductivity ${ }^{10}$, twodimensional (2D) electron gas (2DEG) $)^{11,12}$, ferroelectricity ${ }^{13}$ and blue luminescence ${ }^{14}$, indicating versatile interplay of charge, spin, orbital and lattice degree of freedom ${ }^{15}$. However, previous studies give controversial information about the carrier type in $\mathrm{SrTiO}_{3}$ (see, for example, refs 16,17 and references therein), hampering the thorough distinction of these internal interplay. Recently, ARPES study on FeSe film grown on $\mathrm{SrTiO}_{3}$ has revealed the enhancement of superconducting transition temperature $T_{\mathrm{c}}$ due to the coupling between FeSe electron and oxygen optical phonon in $\mathrm{SrTiO}_{3}$ (ref. 6).

In this article, by examining the surface electronic structure of vacuum-annealed $\mathrm{SrTiO}_{3}$, we report the observation of $2 \mathrm{D}$ liquid of Fröhlich polaron, a new quasi-particle formed by conduction band electron coupled with one polar longitudinal optical (LO) phonon mode. By controlling the annealing temperature, we can precisely tune the surface oxygen vacancy concentration, which develops from 2D to 3D distribution of charges. Moreover, by compiling partial electron yield (PEY) and total fluorescence yield (TFY) XAS spectrum, we are able to identify unambiguously these two distinctive distributions of oxygen vacancies. In the $2 \mathrm{D}$ case, oxygen defect dipoles break the inversion symmetry and initiate the coupling between electrons and polar LO phonons. This intermediate coupling leads to the formation of a new type of quasi-particles, Fröhlich polarons, whose spectra function contains multiple replicas of original bands, equally spaced $\omega_{\mathrm{LO}}$ apart, with $\omega_{\mathrm{LO}}$ the effective energy of LO phonons, as directly observed by ARPES here. Photon-energy-dependent ARPES measurements reveal the 2D nature of Fröhlich polarons. In the $3 \mathrm{D}$ oxygen vacancy case, the $2 \mathrm{D}$ polarons collapse into $3 \mathrm{D}$ electron liquid due to the reversal of symmetry. Our findings reveal the essential role of surface/interface LO-phonon-related large (bi)polarons in understanding superconductivity in perovskite oxides, as well as in high-temperature superconductors.

\section{Results}

Tunable oxygen vacancy concentration via vacuum annealing. Intrinsic $\mathrm{SrTiO}_{3}$ is a band insulator with a $\sim 3.2 \mathrm{eV}$ gap, and experiences a second-order phase transition from cubic to tetragonal structure at the critical temperature of about $105 \mathrm{~K}$ (ref. 18). For the present study, both the XAS and ARPES measurements were performed $\sim 170 \mathrm{~K}$ while the low-energy-electrondiffraction (LEED) patterns were taken at room temperature.
Thus for our study, the bulk $\mathrm{SrTiO}_{3}$ is in the centre-symmetric equilibrium structure, that is, the Ti atom lying in the centre of the regular octahedron of oxygen atoms in the cubic perovskite structure (Fig. 1a). All the experiment data is from the (001) surface as shown in Fig. 1b.

To start with, we present the precisely regulation of surface carriers by controlling the amount of oxygen vacancies via vacuum annealing. We prove the existence of oxygen vacancies by reproducing the well-known LEED patterns (Fig. 2a and also see, for example, ref. 19 and references in), which reflect the surface reconstruction due to the alignment of oxygen vacancies. Charge transfer from oxygen vacancies to $\mathrm{Ti}$ atoms (consequently, $\mathrm{Ti}^{4+}$ to $\mathrm{Ti}^{3+}$ ) allow us to derive the oxygen vacancy concentration [ $\left.V_{\mathrm{O}}\right]$ from the XAS spectra of Ti $L_{2,3}$ edge, attributed to excitations of $2 P_{3 / 2}$ and $2 P_{1 / 2}$ subshells to unoccupied $t_{2 \mathrm{~g}}$ and $e_{\mathrm{g}}$ states, as shown in Fig. $2 \mathrm{~b}, \mathrm{c}$. As indicated by the black arrow and more clearly shown in the insets, a $460 \mathrm{eV}$ peak gradually increases with annealing temperature. According to the theoretical simulation ${ }^{20}$ (Fig. 2f, top), this peak comes from the $\mathrm{Ti}^{3+} L_{3}$ edge. Since X-ray absorption near-edge spectroscopy is an atomic probe, strongly sensitive to its formal oxidation state and coordination chemistry ${ }^{21}$, we can calculate the $\mathrm{Ti}^{3+} / \mathrm{Ti}^{4+}$ ratio by assuming the linear combination of $\mathrm{Ti}^{4+}$ and $\mathrm{Ti}^{3+}$ signals under the single inelastic electron scattering condition 22,23 . As exemplified in Fig. 2f (bottom) for the PEY XAS spectra from surface annealed at $1,000^{\circ} \mathrm{C}$, the experimental spectra can be decomposed into $85 \% \mathrm{Ti}^{4+}$ and $15 \% \mathrm{Ti}^{3+}$, using multiple leastsquares fit. Figure $2 \mathrm{~g}$ schematically summarizes the fitted PEY ratio corresponding to surfaces with different annealing temperature. The monotonic increase of oxygen vacancies with annealing temperature clearly demonstrates that we can control and reproduce the carrier concentration of the $\mathrm{SrTiO}_{3}$ samples. This point has been further corroborated by the agreement between the $\mathrm{Ti}^{3+} / \mathrm{Ti}^{4+}$ ratio calculated by PEY XAS and Ti 3p core levels shown in the Supplementary Fig. 1. Notice that we have chosen a similar energy window $(\sim 40 \mathrm{eV})$ to collect secondary electrons for XAS spectra and core level photoelectrons spectra, ensuring similar depth sensitivity in both data sets.

In addition, we can also prove the increase of surface oxygen vacancies from the $\mathrm{O} K$-edge XAS spectra (Fig. 2d,e). As shown clearly in the insets, the A2 peak increases dramatically for the surface annealed at $1,200^{\circ} \mathrm{C}$. This peak arises from the Ti-O $p d$ hybridization with a $\sigma$-bonding character. Its increase indicates the lattice distortion caused by oxygen vacancies, in qualitatively agreement with our conclusion based on $\mathrm{Ti} L_{2,3}$-edge data. Given that each oxygen vacancy nominally transfers two electrons to the $\mathrm{Ti} d$ band $^{24}$, and there are three $\mathrm{O}$ atoms but only one Ti atom in the unit cell, $\mathrm{Ti}^{3+}$ ratio is six times the value of the oxygen vacancy concentration $\left[V_{\mathrm{O}}\right]$ (Fig. 2g), which allows XAS on Ti $L_{2,3}$ edge be much more sensitive than $\mathrm{O} K$ edge. Thus it is sensible to calculate the $\left[V_{\mathrm{O}}\right]$ from the XAS spectra of Ti $L_{2,3}$ edge.

More importantly, we show that the oxygen vacancy distribution displays a clear dimensionality change. Figure $2 \mathrm{~g}$ also summarizes $\left[V_{\mathrm{O}}\right]$ values fitted from TFY-XAS spectra. For a given annealing temperature, both PEY and TFY spectra are recorded simultaneously from the same sample. Contrary to the monotonic increase of PEY $\left[V_{\mathrm{O}}\right]$, the TFY concentration remains at noise level at the beginning and only increases with annealing temperature above $1,000^{\circ} \mathrm{C}$. This distinctive behaviour is based on the different depth sensitivity of PEY and TFY spectra ${ }^{25}$. The PEY signals, that is, energy-selected secondary electrons in present case, typically from an average depth of $<1 \mathrm{~nm}$ (approximately two unit cells deep), are highly surface sensitive. In contrast, the TFY signals mainly give bulk information due to the long mean free path (MFP) of photons, $(\sim 100 \mathrm{~nm})$. Thus, we 


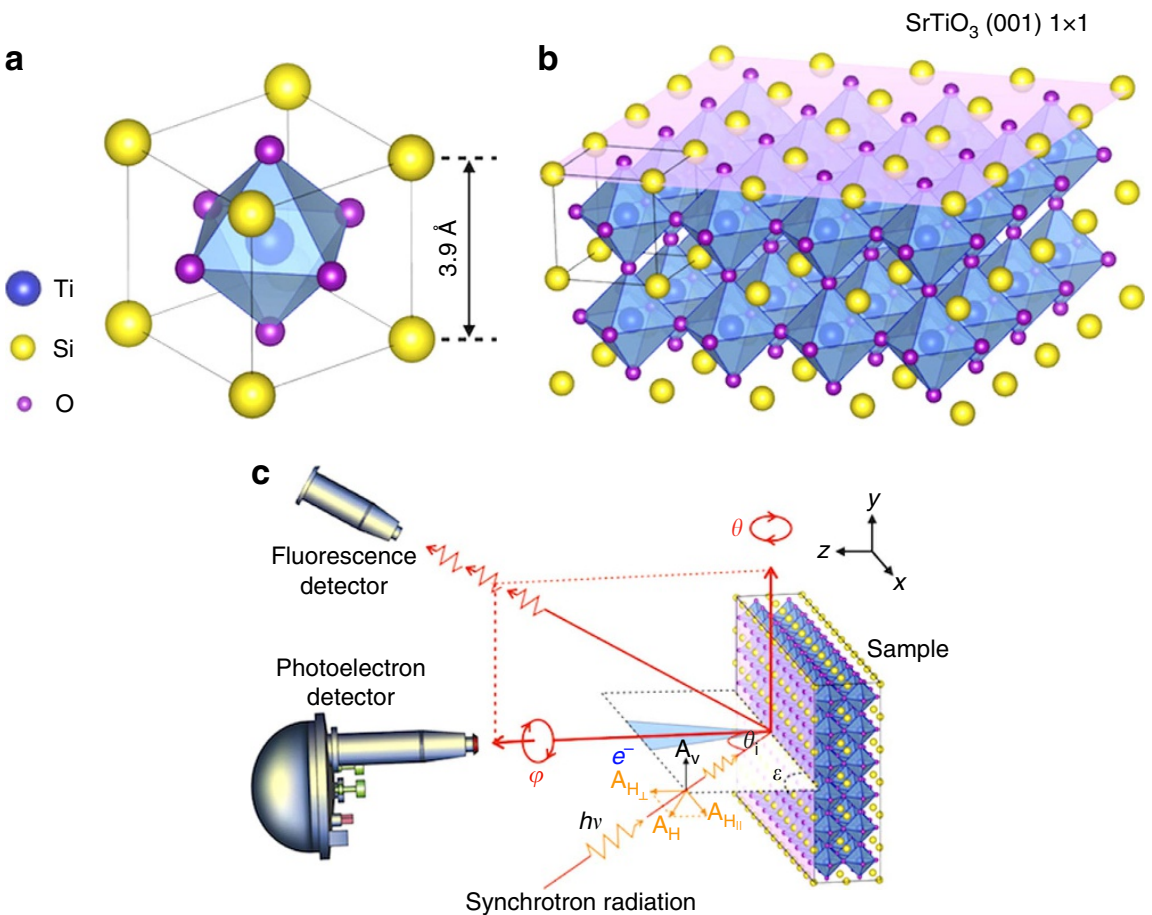

Figure 1 | Lattice structure of $\mathrm{SrTiO}_{3}$ and measurement geometry. (a) The cubic unit cell, with the $\mathrm{TiO}_{6}$ octahedron shown as magenta surfaces. $\mathrm{O}$ atoms at $\mathrm{O} 1$ cites have Ti-O bonds along $z$ direction, while $\mathrm{O} 2$ cites have Ti-O bonds parallel to the $x-y$ plane. (b) A $4 \times 4 \times 2$ block of unit cells. The top layer shown in light purple indicates the (001) surface. (c) Experimental geometry for XAS and ARPES measurements. $\mathbf{A}_{H}\left(\mathbf{A}_{V}\right)$ is the vector potential of the incident light with linear horizontal (vertical) polarization; $\theta$ and $\phi$ denote the polar and azimuthal degree of freedom and $\theta_{\mathrm{i}}$ is the polar angle of incidence. The analyser slits permit photoelectron detection along the $x$ axis. For all different $\theta$, the incident photon momentum, the photoelectron momentum and the surface normal lie on the same plane $(\varepsilon)$. For all the ARPES data, we set the $\theta_{\mathrm{i}} \sim 75^{\circ}$, very close to the grazing angle, to enhance the surface contribution. We use linear horizontal polarization (LH). The beam polarization is even to the scattering plane. According to the matrix element effect (MEE), only orbitals with even symmetry respect to the scattering plane can be excited and detected.

a

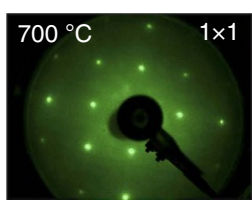

b

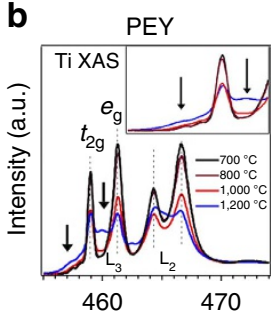

Photon energy (eV)
$4.6 \times 10^{13} \mathrm{~cm}^{-2}$
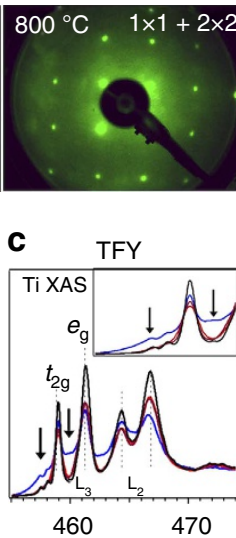

Photon energy (eV)
$1.0 \times 10^{14} \mathrm{~cm}^{-2}$
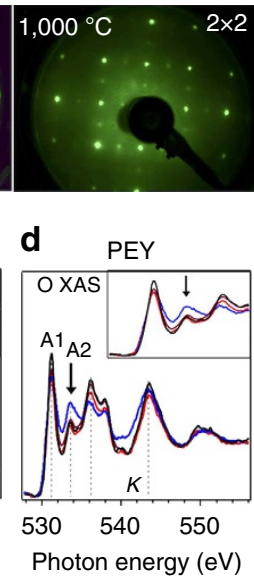

$3.6 \times 10^{21} \mathrm{~cm}^{-3}$
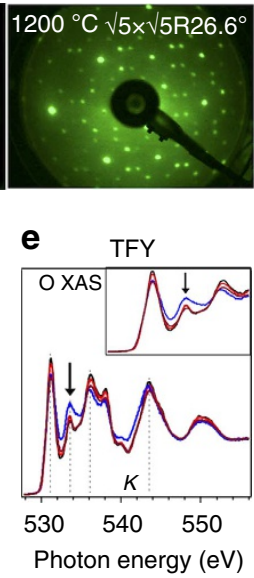

f

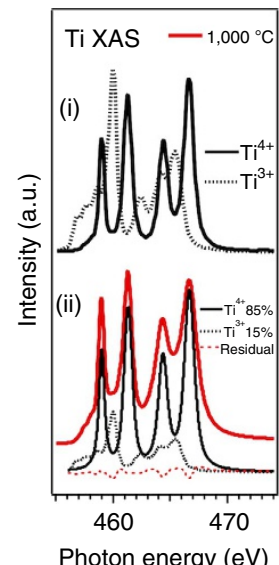

g

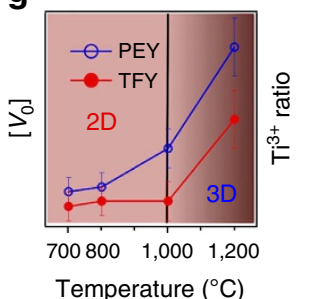

Temperature $\left({ }^{\circ} \mathrm{C}\right)$

Figure 2 | Creating 2D and 3D oxygen vacancies. (a) LEED patterns with surface reconstruction labelled for $\mathrm{SrTiO}_{3}$ surfaces prepared at different annealing temperature sequentially. The corresponding carrier densities are calculated by $\mathrm{Ti}^{3+}$ ratio from $\mathrm{PEY} X \mathrm{XS}$ spectra for $2 \mathrm{D}$ case $\left(\leq 1,000{ }^{\circ} \mathrm{C}\right)$ and TFY-XAS spectra for $3 \mathrm{D}$ case $\left(1,200^{\circ} \mathrm{C}\right)$. See Methods for details. (b-e) Ti $L_{2,3}$-edge and $\mathrm{O} K$-edge XAS spectra from different surfaces. Black arrows indicate the peak intensity coming from $\mathrm{Ti}^{3+}$. (f) Top, numerically calculated XAS spectra of $\mathrm{Ti}^{3+}$ and $\mathrm{Ti}^{4+}$ ions in $\mathrm{SrTiO}_{3}$. Bottom, decomposition of experimental XAS spectra, exemplified by the PEY spectra for surfaces annealed at $1,000^{\circ} \mathrm{C}$. (g) Schematic evolution of oxygen vacancy concentration and $\mathrm{Ti}^{3+}$ ratio from PEY and TFY spectra. Vertical black line indicates the boundary between $2 \mathrm{D}$ and $3 \mathrm{D}$ oxygen vacancies.

conclude that vacuum annealing from $800^{\circ} \mathrm{C}$ to $1,000^{\circ} \mathrm{C}$ generates $2 \mathrm{D}$ oxygen vacancies confined at the surface. However, for samples annealed above $1,000^{\circ} \mathrm{C}$, the high concentration of oxygen vacancies presents a $3 \mathrm{D}$ distribution of charges. For the whole annealing temperature range, the concentration of induced surface charge carriers (2D and 3D) increases monotonously with temperature. In addition, judged from the sharp LEED pattern, the vacancies are well-aligned. 
These ordered defect dipoles break the local inversion symmetry, allow the Raman measurement of first-order phonons ${ }^{26}$ and suggest a phase with ferroelectric-like polarization at the reduced surface of $\mathrm{SrTiO}_{3}$ (ref. 27).

Observation of Fröhlich polaron spectra by ARPES. It is natural to ask what kind of electronic structure does this ferroelectric-like layer correspond to. Figure $3 \mathrm{a}$ shows the symmetrized Fermi surfaces (FSs) from the $\mathrm{SrTiO}_{3}$ surface annealed at $700{ }^{\circ} \mathrm{C}$. Thanks to the matrix element effect, we can selectively excite electrons with different orbital symmetry (see Fig. 1c for measurement geometry). No ARPES spectral intensity can be detected at the first Brillouin zone centre ( $\Gamma$ and $\Gamma_{00}$ specifically). FSs at $\Gamma_{10}$ and $\Gamma_{01}$ are elliptical, with $d_{x z}$ (Fig. 3f) and $d_{y z}$ orbital character, respectively. The circular FS at $\Gamma_{11}$ are from $d_{x y}$ orbital (Fig. 3b). Fitted dispersions (black dashed lines in Fig. 3b,c,f) show an effective mass of $\sim 0.6 m_{\mathrm{e}}$ ( $m_{\mathrm{e}}$ the free electron mass) for $d_{x y}$ band, and $\sim 6 m_{\mathrm{e}}$ for $d_{x z}$ band (see Supplementary Fig. 2 for fitting details). These lighter effective masses will contribute to the high carrier mobility ${ }^{12,28}$.

An extraordinary distinction is that there exist replica bands for each orbital (Fig. 3b,f), lying $\sim 90 \mathrm{meV}$ higher than the original, which can be clearly distinguished from the energysecond-derivative spectra in Fig. 3c. The replica forms one more higher-lying electron pocket, as shown by the constant-energy contours in Fig. 3e. These replicas are attributed to the many-body effect, specifically, the electron-phonon coupling
$(\mathrm{EPC})^{6,29}$. The characteristic energy, $\sim 90 \mathrm{meV}$, corresponds to one surface polar LO phonon mode ${ }^{30}$. These dispersions hence describe electrons weakly coupled to a lattice vibrational mode by the Coulomb interaction, which together can move coherently through the solid and composite a new type of quasi-particle, that is, large polaron. We use the weak-coupling field-theoretical Fröhlich Hamiltonian ${ }^{8}$ as the first approximation to describe EPC in both $3 \mathrm{D}$ and $2 \mathrm{D}$ cases:

$$
H=\frac{\mathbf{p}^{2}}{2 m_{\mathrm{b}}}+\sum_{\mathbf{k} j} \hbar \omega_{\mathrm{LO}, j} a_{\mathbf{k} j}^{\dagger} a_{\mathbf{k} j}+\sum_{\mathbf{k} j}\left(V_{\mathbf{k}, j} e^{i \mathbf{k} \vec{r}} a_{\mathbf{k}_{j}}+\text { h.c. }\right)
$$

Where $\mathbf{r}$ is the position coordinate operator of the electron with band mass $m_{\mathrm{b}}, \mathbf{p}$ is its canonically conjugate momentum operator; $a_{\mathbf{k} j}^{\dagger}\left(a_{\mathbf{k} j}\right)$ is the creation (annihilation) operator for the $j$-th LO phonons of wave vector $\mathbf{k}$ and energy $\hbar \omega_{\mathrm{LO}, j}$. The $V_{\mathbf{k} j}$ is the Fourier component of the EP interaction. Considering one branch of dispersion-less LO-phonon (Einstein model) ${ }^{31-33}$, this interaction system becomes one of the exactly solvable models in many-body physics ${ }^{34}$. The spectra can be well-described by a Franck-Condon model with Poisson distribution:

$$
\frac{I_{l}}{I_{0}}=\frac{g^{l}}{l !}
$$

Here $I_{0}$ is the quasi-particle peak intensity and $I_{l}$ the $l$-th replica intensity with $l$ phonon(s) dressed. $g$ is a constant, related with the EP interaction in $3 \mathrm{D}$. a

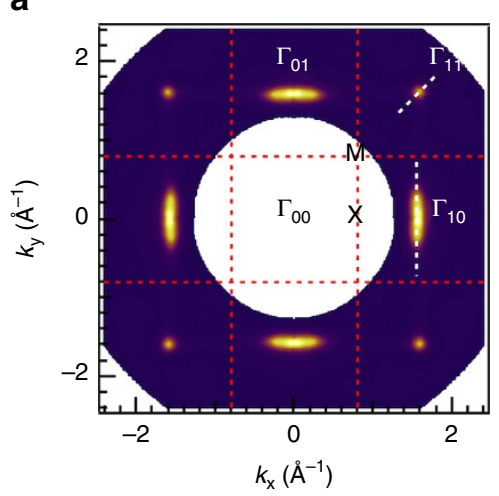

e

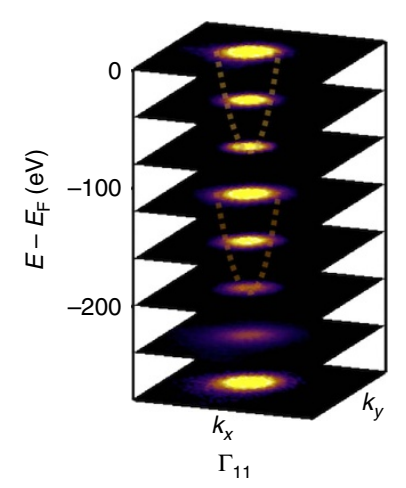

b

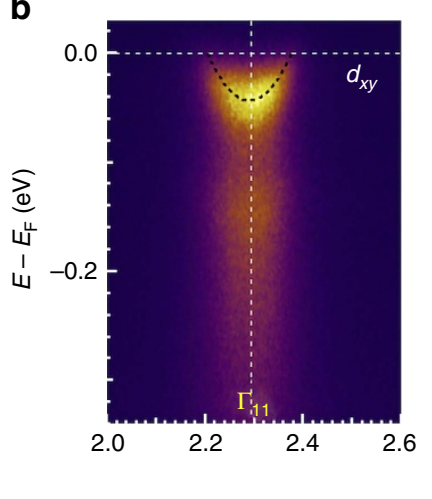

f

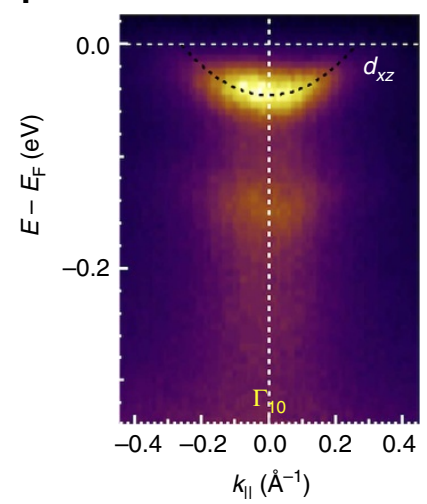

C

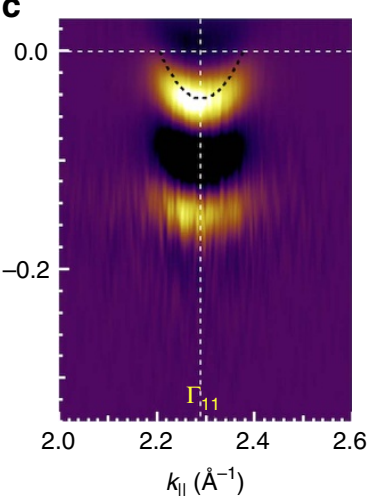

g

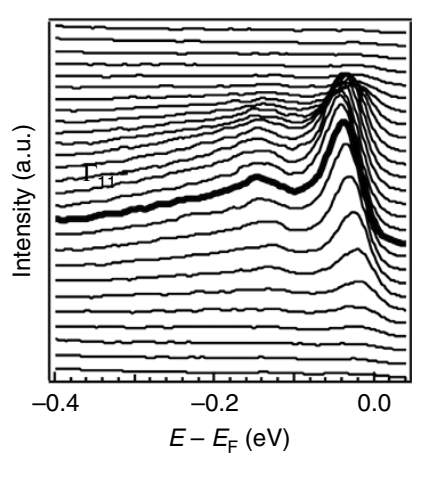

d

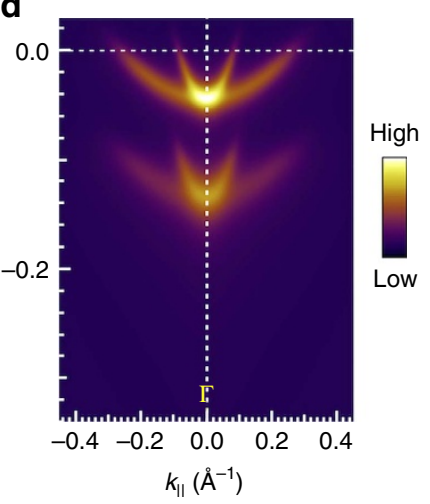

h

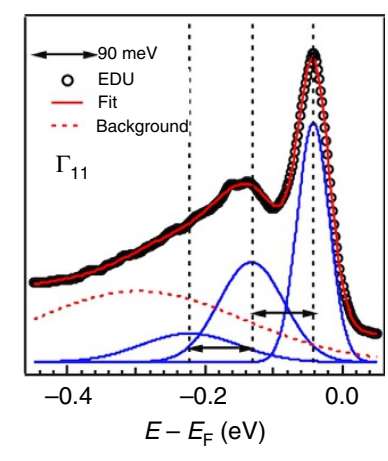

Figure 3 | Observation of Fröhlich polaron spectra by ARPES at $\mathrm{SrTiO}_{3}$ surface prepared at $\mathbf{7 0 0}{ }^{\circ} \mathbf{C}$. (a) Symmetrized Fermi surface of $\mathrm{SrTiO}_{3}$ in $k_{x}-k_{y}$ space. $\Gamma_{10}=2 \pi / a(1,0) \sim(1.61,0) \AA^{-1}$. (b) Dispersion from $\Gamma_{11}$ along [11] direction with $d_{x y}$ orbital character. Dispersion from $\Gamma_{10}$ along [01] direction with $d_{x z}$ orbital character is shown in $\mathbf{f}$. The momentum cuts are shown in dashed while lines. (c) Energy-second-derivative image of spectra in $\mathbf{b}$. (d) Simulated large polaron dispersions with $d_{x y} / d_{y z}$ and $d_{x z}$ orbital characters. (e) Constant-energy contours of large polaron electronic structure, characterized by two (or more) similar electron pockets, indicated by dashed lines. (g) EDC stacks of spectra in $\mathbf{b}$. Black empty circles indicate the EDC shown in $\mathbf{h}$. (h) Franck-Condon line shape fitting. The fitted EDC (red line) is composed of three Gaussian peaks (blue line), separated in energy by 90 meV, and a hump-like background (red dashed line). Black dashed lines in $\mathbf{b}, \mathbf{c}$ and $\mathbf{f}$ are fitted dispersions for the zero-order bands. See Supplementary Fig. 2 for details. 
Considering the ARPES spectra in Fig. 3b,f, the lower-lying band corresponds to charge carriers without dressing phonons, while the higher one corresponds to charge carriers dressing one phonon. Other higher-order bands are nearly invisible but can be deduced from the energy distribution curves (EDCs) fitted with Franck-Condon line shape as shown in Fig. 3h (refs 6,29). The fitting gives an energy separation $\omega_{\mathrm{LO}} \sim 90 \mathrm{meV}$ and $g=0.85$. We can also use other fitting parameters $\alpha_{3 \mathrm{D}}$ and $\alpha_{2 \mathrm{D}}$, to characterize the Fröhlich coupling constant. Inferred from the zero-phonon peak intensity fraction of total weight, according to the diagrammatic quantum Monte Carlo simulation ${ }^{35}$, we have $\alpha_{3 \mathrm{D}} \sim 2.5$. Using the scaling relation $Z_{2 \mathrm{D}}(\alpha)=Z_{3 \mathrm{D}}(3 \pi \alpha / 4)$ for $2 \mathrm{D}$ polarons ${ }^{36}$, we have $\alpha_{2 \mathrm{D}} \sim 1.1$. Notably, here we use a common and available approach to estimate the $2 \mathrm{D}$ coupling strength indirectly. However, although beyond the scheme of present work, a more accurate model directly describing $2 \mathrm{D}$ Fröhlich polaron spectra is highly desired.

It is worthy to note that the whole spectra with multiple bands we observed here describe the ground state of Fröhlich polaron, not the excited state, since the temperature is set to zero for the theoretical spectra function ${ }^{34}$. In the ground state of Fröhlich polaron, some probability exists that the charge carriers have different set of energy $\omega_{l}=\varepsilon_{b}-\Delta+\omega_{\mathrm{LO}} l$. Furthermore, this is different from the characteristic 'peak-dip-hump' spectra in cuprates $^{37,38}$, in which case localized small polarons show no high-order dispersion. Our discovery only becomes possible because of the intermediate coupling strength. The discrete phonon mode energy is bigger than the electron bandwidth and most crucially, because the forward scattering is dominating the EPC, as discussed in the recent literature. Figure $3 \mathrm{~d}$ shows the simulated dispersion of Fröhlich polaron for both $d_{x y}$ and $d_{x z y z}$ orbitals, with the full width at half maximum (FWHM) set as constant, $25 \mathrm{meV}$ and without multiple phonon contribution. Our discovery represents a 'rare' field theoretical model of Fröhlich polaron, which is exactly solvable ${ }^{29,34}$.

One may wonder if these multiple bands arise from band bending effect due to the quantum confinement of the conduction band bottom. In fact, the 2D electron gases/liquids at $\mathrm{SrTiO}_{3}$ surface have become model systems for engineering emergent behaviour and studying the quasi-particle dynamics in complex transition metal oxides ${ }^{11,12,15}$. Here we rule out this possibility due to the fact that the sub-bands in the quantum confinement picture are real electronic structure, crossing Fermi level, but our observed shadow bands are the 'echo' of EPC, without Fermi level crossing. See Supplementary Fig. 3 for schematic understanding. We also clarify that, contrary to previous works ${ }^{12,39}$, during our experiments, all the surfaces displayed no detectable dependence on synchrotron irradiation, as demonstrated by the core level and XAS spectra in Supplementary Fig. 4.

Many seminal studies have been recently done describing the 2DEG scenario, which provide a lot of information even if many important aspects of this phenomenon are still under inflamed discussion. What is important, however, is that there is a quite robust consensus on the conditions in which the 2DEG is stable. Consequently, we have been able to identify three important parameters: carrier density, oxygen vacancy concentration and thickness of the surface layer where the 2DEG takes place. The reported value for the carrier density is close to $10^{14} \mathrm{~cm}^{-2}$ (see, for example, refs 11,15). For the oxygen vacancies, most of the works report a rather constant concentration ${ }^{11,12,40}$. Finally, the thickness is estimated in most of the important works around four to eight cell units ${ }^{11,12,15,40}$. For the 'polaron' scenario, however, the formation appears when the carrier density, the oxygen vacancies and the thickness of the surface layer are in rather different ranges. In our manuscript, we have demonstrated that the polarons only form when the carrier density is close to
$10^{13} \mathrm{~cm}^{-2}, 1$ order of magnitude lower than that for the case of 2DEG. For the oxygen concentration, the polarons appear for approximately half the concentration of oxygen vacancies reported for 2DEG systems (Supplementary Fig. 1). Finally, for the thickness, thanks to the parallel detection of XAS and ARPES we can confirm that the thickness of the surface polarons is not larger than one to two cell units, at least four to eight times smaller than that for the 2DEG surface layer.

Dimensionality and stability of the Fröhlich polarons. As deduced from the XAS spectra, annealing below $1000^{\circ} \mathrm{C}$ creates 2D charge carrier localized mainly at the surface. From ARPES spectra we identify these charge carriers as Fröhlich polarons. We then directly demonstrate the 2D nature of Fröhlich polarons by ARPES. Photon-energy-dependent ARPES can map the FS at 3D momentum space (see Methods). Figure $4 \mathrm{a}$ shows the FS of $d_{x y}$ orbital around one bulk $\Gamma$ point in $k_{x}-k_{y}-k_{z}$ momentum space from surface annealed at $800^{\circ} \mathrm{C}$. To highlight the detail, we plot the second-derivative photoemission intensity versus momentum. Its cylindrical shape and the lack of dispersion along $k_{z}$ direction directly confirm the $2 \mathrm{D}$ nature of the FS. The projection of this 2D FS onto $k_{x}-k_{y}$ momentum space composes an open circle. On the contrary, for surface annealed at $1,200{ }^{\circ} \mathrm{C}$, the corresponding $k_{z}$ dispersion of the FS shows a ellipsoid-like shape, whose projection shows filled circle feature (Fig. 4d), composing evidence of 3D carriers. Consequently, the EDCs analysis as a function of the annealing temperature (Fig. 4c) shows the polaronic bands till the oxygen vacancies present a $3 \mathrm{D}$ distribution. In fact, only one Gaussian peak could be fitted to the $1,200^{\circ} \mathrm{C} \mathrm{EDC}$, with a non-vanishing background connecting the oxygen vacancy band (Supplementary Fig. 1c). This higher binding energy spectral weight commonly results from electron interaction, reminiscent of liquid behaviour ${ }^{12}$. Thus, both FSs and EDCs spectral analysis prove the 2D nature of Fröhlich polarons. The transition from 2D Fröhlich polaron to 3D electron liquid will be discussed in detail later.

Despite its ultimate collapse, 2D Fröhlich polarons are relatively stable over a wide carrier density. The carrier density can be calculated from the Luttinger area of the FSs (see Methods), shown in Fig. 5a,b (see Supplementary Figs 5 and 2 for detailed FSs and fitting). For 2D Fröhlich polaron, we have $n_{2 \mathrm{D}} \sim 5.7 \times 10^{13} \mathrm{~cm}^{-2}, 7.4 \times 10^{13} \mathrm{~cm}^{-2}$ and $7.6 \times 10^{13} \mathrm{~cm}^{-2}$ for surfaces prepared at $700^{\circ} \mathrm{C}, 800^{\circ} \mathrm{C}$ and $1,000{ }^{\circ} \mathrm{C}$, respectively. We have also estimated the carrier density according to the $\mathrm{Ti}^{3+}$ $/ \mathrm{Ti}^{4+}$ ratio from PEY XAS spectra (see Methods). For $2 \mathrm{D}$ case, we got $n_{2 \mathrm{D}} \sim 4.0 \times 10^{13}, 4.6 \times 10^{13}$ and $1.0 \times 10^{14} \mathrm{~cm}^{-2}$ for corresponding surfaces, in qualitative agreement with ARPES data. It is worthy to note that, for both ARPES and XAS, we assume an ideal 2D scenario. In reality the probe depth for our ARPES and XAS measurements are different since the photoelectron kinetic energy is about $95 \mathrm{eV}$ for ARPES and $40 \mathrm{eV}$ for XAS. As shown in Fig. 4c, from all samples annealed between $800^{\circ} \mathrm{C}$ and $1,000^{\circ} \mathrm{C}$, Franck-Condon line shape fitting gave similar second and third order polaronic peak weight, indicating a constant EPC and relatively stable polarons, regardless of the vacancies organization or charge concentration.

Estimated from the $2 \mathrm{D}$ carrier density for $700{ }^{\circ} \mathrm{C}$ case, the Wigner-Seitz radius $r_{\mathrm{s}}=\left(2 \pi n_{2 \mathrm{D}}\right)^{-1 / 2}$ is $\sim 5.3 \AA$ and the average separation between two Fröhlich polarons is $\sim 11 \AA$ (approximately three unit cells). Consequently, the momentum width $\Delta k$ from ARPES spectra at Fermi momentum gives a polaron MFP $\lambda \sim 10 \AA$. The Franck-Condon fitting in Fig. $4 \mathrm{c}$ also gave similar hump-like background for all the three surfaces. Note that, unlike the $1,200^{\circ} \mathrm{C}$ case with oxygen vacancy band observed at higher binding energy, this background can only originate from the 
a

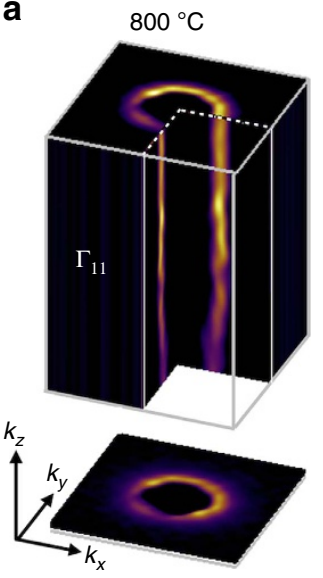

b

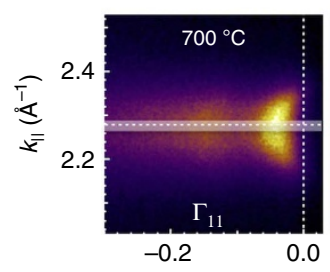

Low

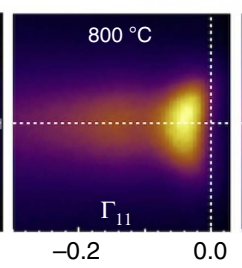

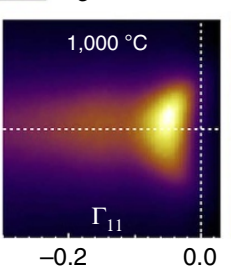

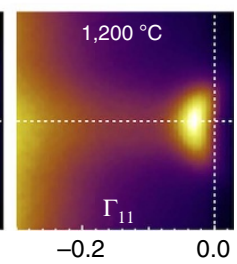

C
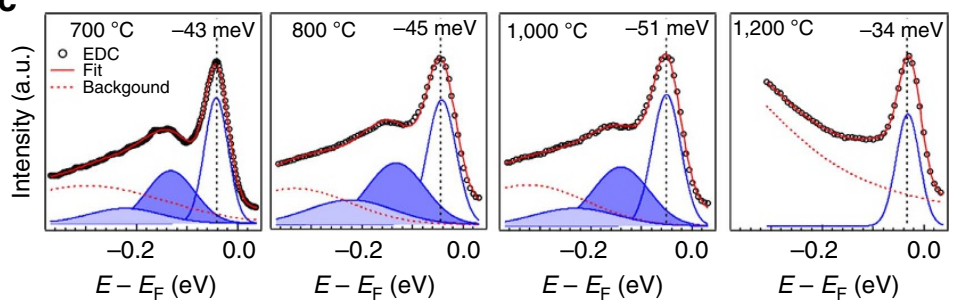

d

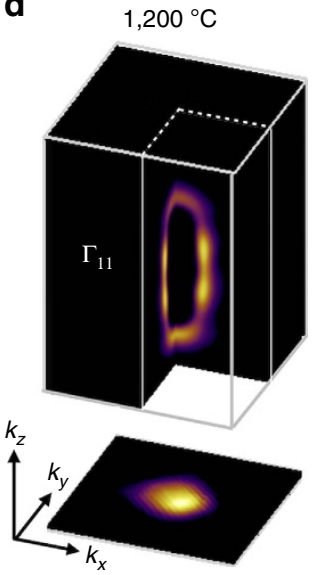

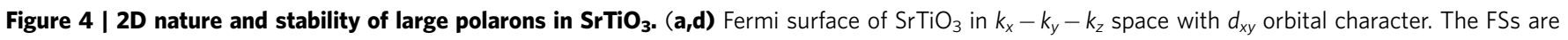

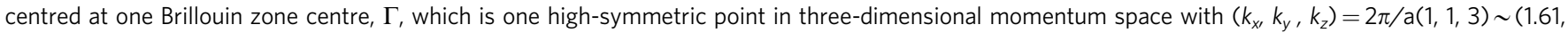

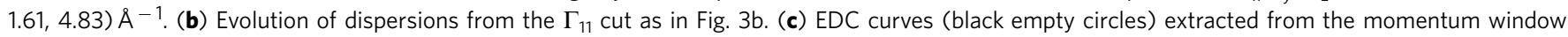
shown in grey in $\mathbf{b}$. Franck-Condon line shape fitted curves are shown with red lines. Blue lines represent individual Gaussian peaks.
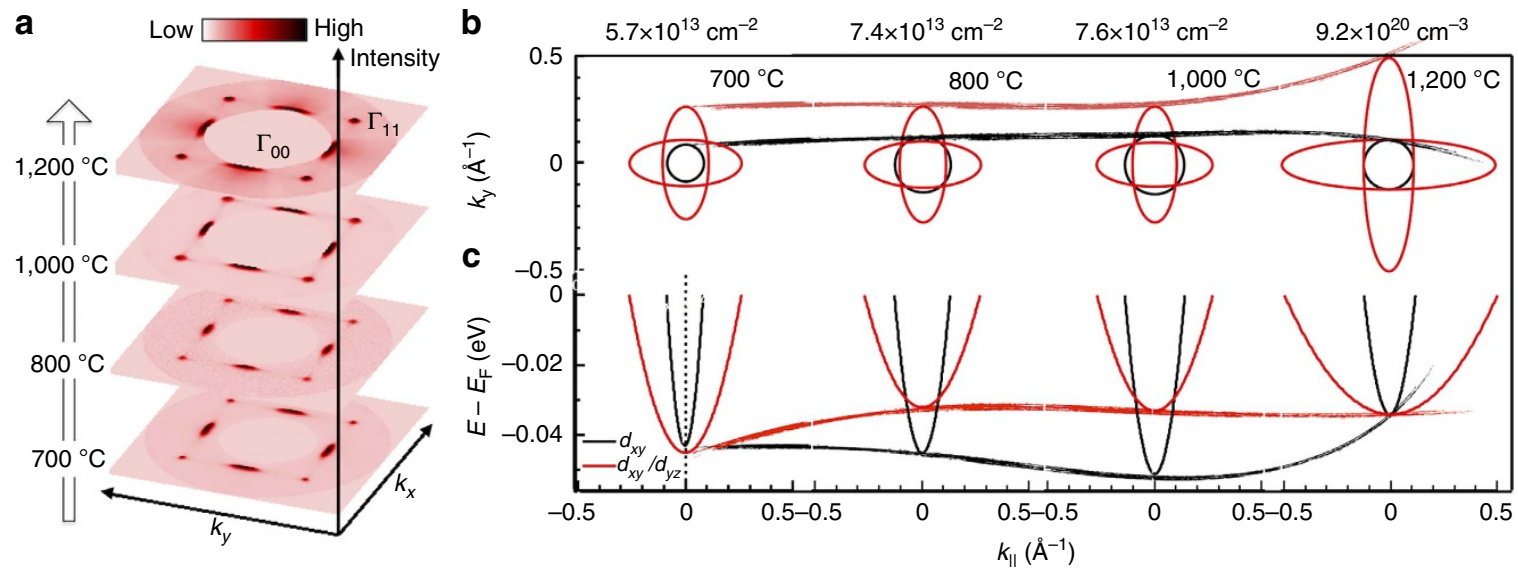

Figure 5 | Evolution of Fermi surfaces and dispersions. (a) Symmetrized Fermi surfaces of $\mathrm{SrTiO}_{3}$ in $k_{x}-k_{y} \mathrm{space}_{\mathrm{for}}(001$ ) surfaces annealed at different temperature. The raw data is shown in Supplementary Fig. 5. (b) Fitted FSs for all the three orbitals. The calculated carrier densities are shown for corresponding FSs. See Methods for calculation formula. The superimposed red and black curves indicate the size change for $d_{x z}$ FS and $d_{x y}$ FS, respectively. (c) Fitted dispersions for different orbitals. See Supplementary Fig. 2 for fitting details. The superimposed red and black curves indicate the band bottom change for $d_{x z}$ band and $d_{x y}$ band (zero order), respectively.

conduction band, and most likely, attributed to the interaction between polarons. In addition, the effective mass for Fröhlich polaron ( $\sim 0.6 m_{\mathrm{e}}$ for $d_{x y}$ orbital) is even lighter than that both for $3 \mathrm{D}$ liquid in our case $\left(\sim 1.1 \mathrm{~m}_{\mathrm{e}}\right)$ and for $2 \mathrm{D}$ electron gas from reported data $\left(\sim 0.7 m_{\mathrm{e}}\right)^{11}$, indicating its high mobility. Thus it is proper to conclude that $2 \mathrm{D}$ liquid of Fröhlich polarons with high mobility can be created and confined at the surface of $\mathrm{SrTiO}_{3}$.

\section{Discussion}

The ultimate collapse of $2 \mathrm{D}$ Fröhlich polaron to $3 \mathrm{D}$ liquid is puzzling and deserves further examination. Here we interpret it exclusively from the point of view of symmetry. Considering that the EPC is local and linear, in lattice with space inversion symmetry, only phonons with even parity can contribute to the EPC. The linear contribution from polar LO phonons becomes possible only with inducing symmetry breaking. Intrinsic $\mathrm{SrTiO}_{3}$ has a centre-symmetric structure, with $d_{x y}$ and $d_{x z / y z}$ orbitals degenerate at the bottom of conduction band ${ }^{41}$. As annealed from $800^{\circ} \mathrm{C}$ to $1,000^{\circ} \mathrm{C}$, the oxygen vacancies mainly locate at the $\mathrm{O} 1$ site (Fig. 1a). These highly restricted $2 \mathrm{D}$ defect dipoles break the inversion symmetry along $z$ direction, resulting in the level splitting between $d_{x y}$ and $d_{x z}$ orbitals as shown in Fig. $5 c$. The sign change of splitting between $700{ }^{\circ} \mathrm{C}$ and $800^{\circ} \mathrm{C}$ cases may attribute to the alignment and correlation of dipolar moment. Nevertheless, Franck-Condon line shape fitting yields similar phonon energy and coupling strength (Fig. 4c), indicating the robustness of Fröhlich polaron with broken inversion symmetry. In the case of $1,200^{\circ} \mathrm{C}$, oxygen vacancies expand to $3 \mathrm{D}$ and start to occupy $\mathrm{O} 2$ sites. These $3 \mathrm{D}$ defect dipoles generate relatively even potential along all the three directions. Thus somehow the surface lattices regain its symmetry, deduced by the re-degenerate orbital bands (see Fig. $5 \mathrm{c}$ for $1,200^{\circ} \mathrm{C}$ case). Consequently, EPC fails, and the many-body effect is dominated by electron-electron 
interaction, forming 3D liquid. Our scenario suggests that, besides vacuum annealing, other symmetry-breaking alternatives such as applying electric field ${ }^{42}$ or capping layer ${ }^{6,43}$ may also initiate the electron-LO phonon coupling and create Fröhlich polarons.

The 2D Fröhlich polaron density falls in the range of which superconductivity in perovskite oxide interfaces is observed ${ }^{42,44}$. It has been long proposed that a Bose-Einstein condensation of 2D bipolarons into a superconducting fluid be responsible for the high $T_{\mathrm{c}}$ in cuprates with multiple LO-phonon branches ${ }^{45}$. The recent discovery of high $T_{\mathrm{c}}$ in single unit cell thick iron selenide film (1UC FeSe) grown on $\mathrm{SrTiO}_{3}$ substrate also suggests the intimate interplay between charge carriers in FeSe and LOphonon in $\mathrm{SrTiO}_{3}$ (refs 6,46,47). The transport measurements ${ }^{48}$ first confirm the very high- $T_{\mathrm{c}}$ superconductivity in single layer FeSe/STO system, in agreement with ref. 6. So if one considers these works together, it would be direct to deduce that this EPC is intrinsic for $\mathrm{SrTiO}_{3}$ and be responsible for the enhanced superconductivity in FeSe/STO system. However, even if there are several articles suggesting that the EPC of the $\mathrm{SrTiO}_{3}$ may be relevant in the mechanism of the high- $T_{\mathrm{c}}$ superconductivity, our manuscript is not dealing directly with this issue, in consequence further studies should be carried out to prove or disprove the relation between these two processes.

It is worthy to note that the phonon mode we observed here may not necessarily represent only one LO-branch, but could correspond to the approximation of multiple LO-phonon branches ${ }^{49}$. In this case, the calculated effective coupling constant $\alpha_{3 \mathrm{D}}$ is $2.34\left(m^{*}\right)^{1 / 2}$. Using the band effective mass $\left(m^{*}\right)$ of $d_{x y}$ orbital in 3D liquid case, we have $\alpha_{3 \mathrm{D}} \sim 2.45$, in excellent agreement with our experimental value. In this approximation, large bipolarons are found to have an extended stability region at low temperature. Thus our observation of Fröhlich polaron liquid in $\mathrm{SrTiO}_{3}$ suggests significant relevance of large (bi)polaron for superconductivity and should stimulate broad interest in exploring polaron behaviour in a wide range of perovskiterelated materials, especially high- $T_{\mathrm{c}}$ cuprates and iron-based superconducting film on $\mathrm{SrTiO}_{3}$ substrate.

\section{Methods}

Sample preparation. $\mathrm{SrTiO}_{3}(100)$ single crystals (crystal GmbH and SurfaceNet $\mathrm{GmbH}$ ) were mounted on resistive $\mathrm{Si}$ heaters and cleaned by thermal annealing in vacuum conditions. Each sample was sequentially heated up to particular temperatures, and kept for $20 \mathrm{~min}$, till characteristic LEED patterns are stabilized. At all stages of the cleaning procedure, the pressure was $<10^{-9}$ mbar. All samples presented reproducible and stable phases, with a series of typical reconstructions.

X-ray Absorption Spectroscopy (XAS). For the XAS measurements, from the same sample surface secondary electrons were recorded by Scienta energy analyzer, while fluorescence was recorded simultaneously by Bruker detector. XAS spectra simulation was performed by tetrahedral cluster model calculation. The cluster consists of a central $\mathrm{Ti}$ atom and its nearest neighbour ligand atoms, and the model calculation includes not only the full multiplets of $3 d$ electrons but also configuration interaction.

ARPES. The angle and energy resolutions of the ARPES measurements (Scienta $\mathrm{R} 4000$ ) are set as $0.2^{\circ}$ and $\sim 10 \mathrm{meV}$. The photon energy-dependent ARPES experiments were performed with photon energy varying from $70 \mathrm{eV}$ to $115 \mathrm{eV}$. The $k z$ was calculated by $k_{z}=\hbar^{-1}\left(2 m\left(E_{k} \cos ^{2} \theta+V_{0}\right)\right)^{9 / 2}$. The inner potential $V_{0}=12 \mathrm{eV}$. Unless otherwise stated, we used photon energy at $100 \mathrm{eV}$ for normal ARPES measurements. The $2 \mathrm{D}$ and $3 \mathrm{D}$ carrier density can be calculated from the Luttinger area of the FSs. $n_{2 \mathrm{D}}=k_{\mathrm{F}}^{2} / 2 \pi$ and $n_{3 \mathrm{D}}=k_{\mathrm{F}}^{3} / 3 \pi^{2}$. From the $\mathrm{Ti}^{3+}$ ratio, the carrier density can also be calculated by $n_{2 \mathrm{D}}=$ ratio $\times a^{-2}$ and $n_{3 \mathrm{D}}=$ ratio $\times a^{-3}$ with the lattice constant $\alpha=3.9 \AA$.

\section{References}

1. Landau, L. D. On the motion of electrons in a crystal lattice. Phys. Z. Sowjetunion 3, 664-665 (1933).

2. Devreese, J. T. Polarons. Encyclopedia Appl. Phys. 14, 383-409 (1996).

3. Devreese, J. T. \& Alexandre, S. A. Fröhlich polaron and bipolaron: recent developments. Rep. Prog. Phys. 72, 066501 (2009).
4. Alexandrov, A. S. Theory of high temperature superconductivity beyond bcs with realistic Coulomb and Fröhlich interactions. J. Supercond. Nov. Magn. 26, 1313-1317 (2013).

5. Alexandrov, A. S., Samson, J. H. \& Sica, G. High-temperature superconductivity from realistic Coulomb and Frö hlich interactions. Europhys. Lett. 100, 17011 (2012).

6. Lee, J. J. et al. Interfacial mode coupling as the origin of the enhancement of $T_{c}$ in FeSe films on $\mathrm{SrTiO}_{3}$. Nature 515, 245-248 (2014).

7. Jooss, C. et al. Polaron melting and ordering as key mechanisms for colossal resistance effects in manganites. Proc. Natl Acad. Sci. USA 104, 13597-13602 (2007).

8. Fröhlich, H. Electrons in lattice fields. Adv. Phys. 3, 325-361 (1954).

9. Takagi, H. \& Hwang, H. Y. An emergent change of phase for electronics. Science 327, 1601-1602 (2010).

10. Ueno, K. et al. Electric-field-induced superconductivity in an insulator. Nat. Mater. 7, 855-858 (2008).

11. Santander-Syro, A. F. et al. Two-dimensional electron gas with universal subbands at the sur- face of $\mathrm{SrTiO}_{3}$. Nature 469, 189-193 (2011).

12. Meevasana, W. et al. Creation and control of a two-dimensional electron liquid at the bare $\mathrm{SrTiO}_{3}$ surface. Nat. Mater. 10, 114-118 (2011).

13. Haeni, J. H. et al. Room-temperature ferroelectricity in strained $\mathrm{SrTiO}_{3}$. Nature 430, 758-761 (2004).

14. Kan, D. S. et al. Blue-light emission at room temperature from $\mathrm{Ar}^{+}$-irradiated $\mathrm{SrTiO}_{3}$. Nat. Mater. 4, 816-819 (2005).

15. King, P. D. C. et al. Quasiparticle dynamics and spin-orbital texture of the SrTiO3 two-dimensional electron gas. Nat. Commun. 5, 3414 (2014).

16. Devreese, J. T., Klimin, S. N., van Mechelen, J. L. M. \& van der Marel, D Manybody large polaron optical conductivity in $\mathrm{SrTi}_{1-x} \mathrm{Nb}_{x} \mathrm{O}_{3}$. Phys. Rev. B 81, 125119 (2010)

17. Hao, X., Wang, Z., Schmid, M., Diebold, U. \& Franchini, C. Coexistence of trapped and free excess electrons inSrTiO3. Phys. Rev. B 91, 085204 (2015)

18. Wang, F. Y. \& Gupta, K. Phase transformation in the oxides. Metallurgical Transactions 4, 2767-2779 (1973).

19. Lin, Y. et al. The $(2 \times 2)$ reconstructions on the $\mathrm{SrTiO}_{3}(001)$ surface: A combined scanning tunneling microscopy and density functional theory study. Surf. Sci. 605, L51-L55 (2011).

20. Cho, K. et al. X-ray absorption spectroscopy study of diluted magnetic semiconductors: $\mathrm{Zn}_{1-x} \mathrm{M}_{x} \mathrm{Se}(\mathrm{M}=\mathrm{Mn}, \mathrm{Fe}, \mathrm{Co})$ and $\mathrm{Zn}_{1-{ }_{x}} \mathrm{Mn}_{x} \mathrm{Y}(\mathrm{Y}=\mathrm{Se}, \mathrm{Te})$. Phys. Rev. B 63, 155203 (2001).

21. Newville, M. Fundamentals of XAFS. Rev. Mineral. Geochem. 78, 33-74 (2014).

22. Ohtomo, A., Muller, D. A., Grazul, J. L. \& Hwang, H. Y. Artificial chargemodulation in atomic-scale perovskite titanate superlattices. Nature 419, 378-380 (2002).

23. Zhu, G. Z., Radtke, G. \& Botton, G. A. Bonding and structure of a reconstructed (001) surface of $\mathrm{SrTiO}_{3}$ from TEM. Nature 490, 384-387 (2012).

24. Muller, D. A., Nakagawa, N., Ohtomo, A., Grazul, J. L. \& Hwang, H. Y. Atomicscale imaging of nanoengineered oxygen vacancy profiles in $\mathrm{SrTiO}_{3}$. Nature 430, 657-661 (2004).

25. Stö hr, J. NEXAFS Spectroscopy (Springer Berlin Heidelberg, 2010).

26. Hanzig, J. et al. Migration-induced field-stabilized polar phase in strontium titanate single crystals at room temperature. Phys. Rev. B 88, 024104 (2013).

27. Uwe, H., Yamaguchi, H. \& Sakudo, T. Ferroelectric microregion in

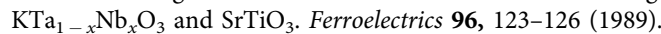

28. Ohtomo, A. \& Hwang, H. Y. A high-mobility electron gas at the $\mathrm{LaAlO}_{3} / \mathrm{SrTiO}_{3}$ heterointer- face. Nature 427, 423-426 (2004).

29. Moser, S. et al. Tunable polaronic conduction in anatase TiO2. Phys. Rev. Lett 110, 196403 (2013)

30. Baden, A. D. et al. Observation of surface optical phonons on $\mathrm{SrTiO}_{3}(100)$. J. Phys. C 14, L1081-L1084 (1981).

31. Fuchs, R. \& Kliewer, K. L. Optical modes of vibration in an ionic crystal slab. Phys. Rev. 140, A2076-A2088 (1965).

32. Wu, X. G., Peeters, F. M. \& Devreese, J. T. Exact and approximate results for the ground-state energy of a Frö hlich polaron in two dimensions. Phys. Rev. B 31, 3420-3426 (1985).

33. Mori, N. \& Ando, T. Electron-optical-phonon interaction in single and double heterostructures. Phys. Rev. B 40, 6175-6188 (1989).

34. Mahan, G. D. Many-Particle Physics (Springer, 2000).

35. Mishchenko, A. S., Prokof ev, N. V., Sakamoto, A. \& Svistunov, B. V. Diagrammatic quantum Monte Carlo study of the Fröhlich polaron. Phys. Rev. B 62, 6317-6336 (2000).

36. Peeters, F. M. \& Devreese, J. T. Scaling relations between the two- and threedimensional polarons for static and dynamical properties. Phys. Rev. B 36, 4442-4445 (1987).

37. Shen, K. M. et al. Missing quasiparticles and the chemical potential puzzle in the doping evolution of the cuprate superconductors. Phys. Rev. Lett. 93, 267002 (2004).

38. Mannella, N. et al. Nodal quasiparticle in pseudogapped colossal magnetoresistive manganites. Nature 438, 474-478 (2005). 
39. Plumb, N. C. et al. Mixed dimensionality of confined conducting electrons in the surface region of $\mathrm{SrTiO}_{3}$. Phys. Rev. Lett. 113, 086801 (2014).

40. McKeown Walker, S. et al. Control of a two-dimensional electron gas onSrTiO ${ }_{3}(111)$ by atomic oxygen. Phys. Rev. Lett. 113, 177601 (2014).

41. Chang, Y. J., Bostwick, A., Kim, Y. S., Horn, K. \& Rotenberg, E. Structure and correlation effects in semiconducting $\mathrm{SrTiO}_{3}$. Phys. Rev. B 81, 235109 (2010).

42. Takahashi, K. S. et al. Local switching of two-dimensional superconductivity using the ferro- electric field effect. Nature 441, 195-198 (2006).

43. Sachs, R., Lin, Z.S. \& Shi, J. Ferroelectric-like SrTiO3 surface dipoles probed by graphene. Sci. Rep. 4, 3657 (2014).

44. Reyren, N. et al. Superconducting interfaces between insulating oxides. Science 317, 1196-1199 (2007).

45. Emin, D. Formation, motion, and high-temperature superconductivity of large bipolarons. Phys. Rev. Lett. 62, 1544-1547 (1989).

46. He, S. et al. Phase diagram and electronic indication of high-temperature superconductivity at $65 \mathrm{~K}$ in single-layer FeSe films. Nat. Mater. 12, 605-610 (2013).

47. Tan, S. et al. Interface-induced superconductivity and strain-dependent spin density waves in $\mathrm{FeSe} / \mathrm{SrTiO}_{3}$ thin films. Nat. Mater. 12, 634-640 (2013).

48. Ge, J. F. et al. Superconductivity above $100 \mathrm{~K}$ in single-layer FeSe films on doped $\mathrm{SrTiO}_{3}$. Nat. Mater. 14, 285-289 (2015).

49. Verbist, G., Peeters, F. M. \& Devreese, J. T. Extended stability region for large bipolarons through interaction with multiple phonon branches. Ferroelectrics 130, 27-34 (1992)

\section{Acknowledgements}

We thank Prof. Z.X. Shen for discussion. The Synchrotron SOLEIL is supported by the Centre National de la Recherche Scientifique (CNRS) and the Commissariat à l'Energie Atomique et aux Energies Alternatives (CEA), France.

\section{Author contributions}

C.C. managed the final data analysis and manuscript writing with initial input from E.F and M.C.A. ARPES and XAS experiments were performed by J.A., E.F. and A.L., and they have also partially participated in the data analysis. M.C.A. has initiated and designed this research. J.A. and M.C.A. have conducted the experimental research programme and data analysis. Also, the manuscript writing was improved by M.C.A. All authors discussed the results and commented on the manuscript.

\section{Additional information}

Supplementary Information accompanies this paper at http://www.nature.com/ naturecommunications

Competing financial interests: The authors declare no competing financial interests.

Reprints and permission information is available online at http://npg.nature.com/ reprintsandpermissions/

How to cite this article: Chen, C. et al. Observation of a two-dimensional liquid of Fröhlich polarons at the bare $\mathrm{SrTiO}_{3}$ surface. Nat. Commun. 6:8585 doi: 10.1038/ncomms9585 (2015).

\footnotetext{
(c) (i)

This work is licensed under a Creative Commons Attribution 4.0 International License. The images or other third party material in this article are included in the article's Creative Commons license, unless indicated otherwise in the credit line; if the material is not included under the Creative Commons license, users will need to obtain permission from the license holder to reproduce the material. To view a copy of this license, visit http://creativecommons.org/licenses/by/4.0/
} 